\title{
Offensive für nutzenorientierte Medizin in Winterthur
}

L. Dubs*

Leistungen für die Patienten optimieren und Kosten in den Griff bekommen: Die Ärztegesellschaft Winterthur-Andelfingen (AWA) sieht einen Ansatz zur Erfüllung dieser Ziele in der nutzenorientierten Medizin, der Evicence-based Medicine (EBM).

Im Weiterbildungsprojekt «EBM Bottom up», das weitgehend durch den Förderverein des Winterthurer Instituts für Gesundheitsökonomie (WIG) an der Zürcher Hochschule Winterthur finanziert wird, eignen sich die AWAMitglieder aller Fachdisziplinen auf freiwilliger Basis die Grundkenntnisse der EBM an.

\footnotetext{
* Dr. Luzi Dubs ist Vorstandsmitglied der Ärztegesellschaft Winterthur-Andelfingen und Präsident des Fördervereins des Winterthurer Instituts für Gesundheitsökonomie.
}

Korrespondenz:

Dr. med. Luzi Dubs

Merkurstrasse 12

CH-8400 Winterthur
Der Begriff der «Evicence-based Medicine» (EBM) ist in aller Munde und löst unterschiedliche Reaktionen inner- und ausserhalb der Ärzteschaft aus. Seit vielen Jahren vermittelt das Winterthurer Institut für Gesundheitsökonomie (WIG) an der Zürcher Hochschule Winterthur, welches ideell und finanziell von einem breitgefächerten Förderverein aus allen Sparten des Gesundheitswesens mitgetragen wird, die methodischen Grundlagen der EBM.

Umfragen haben gezeigt, dass viele Ärztinnen und Ärzte Mühe haben, die Qualität von medizinischen Studien zu beurteilen. Sie empfangen regelmässig Vertreter der Medizinalindustrie und durchschauen oft nicht, wie raffiniert diese ihre Informationen plazieren (siehe Kasten mit typischer EBM-Szene).

\section{EBM nutzbar machen}

Um die Frage zu klären, ob EBM tatsächlich mehr als nur ein neues Schlagwort für Altbewährtes, mehr als eine neue Religion einzelner missionarischer Besserwisser und mehr als eine Metaanalysenakrobatik für Statistiker darstellt, hat der gesamte Vorstand der Ärztegesellschaft Winterthur-Andelfingen im Januar 2003 einen neu gestalteten, eintägigen Intensivkurs absolviert. Aufgrund der Eindrücke beschloss er einstimmig, das Thema der EBM bzw. der eigentlichen Nutzenforschung zum Schwerpunkt der standespolitischen Aktivitäten zu erklären und jedem Mitglied der AWA den Besuch von Kursen mit theoretischen und praktischen Inhalten nahezulegen.
In Zusammenarbeit mit dem WIG entstand das Projekt «EBM Bottom up». Zur Hauptsache wird es mit Sponsorengeldern der 14 im Vorstand des Fördervereins vertretenen Organisationen finanziert, zu welchen auch die AWA und die Ärztegesellschaft des Kantons Zürich (AGZ) gehören. Im Vorstand des Fördervereins ist die Einsicht gereift, dass diese Pilotinitiative der eigenen Schwerpunktsetzung im Bereich «Nutzenforschung» optimal entspricht.

\section{Interesse wecken mit praxisorientierten Kursen}

Das Projekt «EBM Bottom up» beinhaltet nebst der Erstellung eines angepassten Skriptums die Durchführung von eintägigen Intensivkursen und von Übungsnachmittagen mit konkreten Beispielen. Das Schwergewicht wird ganz bewusst auf die Anwendbarkeit in Praxis und Spital gelegt. Im Intensivkurs werden die grundlegenden EBM-Techniken behandelt: Vierfeldertafel, Sensitivität, Spezifität, Krankheitswahrscheinlichkeiten, Wirkungsgrössen wie absolute und relative Risikoreduktion, «number needed to treat», klinische Relevanz usw. Das wahre Gesicht der evidenzbasierten Medizin wird in einen weiten Kontext gestellt. Wissenschaftstheoretische Aspekte, Fragen zu zeitgemässen medizinischen Klassifikationen und allgemeine philosophische Fragestellungen werden dabei diskutiert. Der Kurs fusst auf den seit Jahren bewährten dreitägigen EBM-Kursen des WIG, welche weiterhin im Angebot des Instituts verbleiben.

\section{Wie können die Ergebnisse von EBM gemessen werden?}

Die Zahl der ersten Kursanmeldungen und die Reaktionen der ersten Kursteilnehmer wirken ermutigend und deuten darauf hin, dass das Projekt «EBM Bottom up» gute Erfolgschancen hat. Wie der Erfolg der EBM gemessen und dokumentiert werden kann, wird bereits heute 
intensiv in der Projektleitung diskutiert. Unabdingbare Voraussetzung für den langfristigen Erfolg von EBM ist sicherlich eine entsprechende Geisteshaltung bei den Ärzten, aber auch bei den Patienten. Es gilt, bestehende Schwellenängste zu überwinden und nicht stets das Machbare, sondern das Sinnvolle anzustreben. Ein weiterer, schwierig zu messender Faktor sind die Auswirkungen auf die Kosten. Denn nicht nur die reinen Arztkosten sind einzubeziehen, sondern auch Kosten, die durch Arbeitsausfall oder die Betreuung ausserhalb der Arztpraxis oder des Spitals entstehen. Die Entwicklung von TrustCentern oder ortsansässige Hausarztmodelle wie Wintimed oder HAWA, die sich zunehmend mit Fragen der Budgetverantwortung beschäftigen, könnten die Erfassung der Arztkosten erleichtern.

Die Optimierung des diagnostischen und therapeutischen Aufwandes zu messen, ist einstweilen noch eine ernsthafte Herausforderung. Erhebungen über die Patientenzufriedenheit reichen nicht aus. Grundsätzlich können im Einzelfall weder Patient noch Arzt entscheiden, ob eine Verbesserung bzw. Verschlechterung

\section{Eine typische EBM-Szene}

Pharmavertreter Ledergerber zu Besuch bei Dr. med. Klöti*

Dr. med. Pius Klöti praktiziert seit 15 Jahren als Landarzt, ist dabei zufrieden und empfängt jedes Jahr einmal den Pharmavertreter Ledergerber zwecks Informationen über neueste Medikamente. Ledergerber präsentiert auch anlässlich des aktuellen Besuches neueste Studienergebnisse. Diesmal geht es um die jüngst publizierten Behandlungserfolge bei Osteoporose mit dem neuen CONTRAFRAKT-PLUS. Die Säulendiagramme in der vorgelegten Studienbroschüre zeigen eindrücklich und mühelos eine Reduktion der Schenkelhalsfrakturrate um 33\% nach 10jähriger Einnahme des Medikamentes im Vergleich mit einem Placebo. Während Ledergerber seine ersten Musterpackungen auf dem Tisch ausbreitet, berichtet er mit sichtlichem Stolz über den günstigen Monatspreis von Fr. 78.- für die Behandlung.

Klöti wirkt nachdenklicher als auch schon und schaut sich etwas länger als üblich die Studie an. An der Stelle, wo er die konkreten Zahlen über die Inzidenzen entdeckt, hält er inne. Eine 10jährige Medikamenteneinnahme reduziert die Schenkelhalsfrakturrate von $6 \%$ auf $4 \%$. Rasch gelangt er via Berechnung der absoluten Risikoreduktion zur Number-needed-to-treat von 50. Er müsste also 50 Frauen 10 Jahre lang mit CONTRAFRAKT-PLUS behandeln, um eine einzige Schenkelhalsfraktur zu vermeiden. Bei einem Jahrespreis von 936 Franken belaufen sich die Kosten demnach auf Fr. 468 000.-, um dieses Ziel zu erreichen. Ledergerber hat mit der Angabe der relativen Risikoreduktion von 33\% zwar keine Unwahrheiten verbreitet. Klöti hat sich aber diesmal nicht erwischen lassen.

Warum möchte Dr. Klöti ihn vorläufig nicht mehr empfangen? - fragt sich Ledergerber beim Verlassen der Praxis leicht konsterniert. Dr. Klöti legt die zurückgelassenen Musterpackungen diesmal nicht in den Medikamentenschrank.

(Namen von der regionalen Ärztegesellschaft abgeändert.) wegen oder trotz der Behandlung eingetroffen ist. Umso wichtiger ist die methodische Schulung zur zuverlässigen Literaturinterpretation von Studien mit einem Vergleichskollektiv ohne oder mit einer Alternativbehandlung.

\section{EBM ist ein Kind unserer Zeit}

Da in unserer hochentwickelten Medizin die erwarteten Ergebnisunterschiede immer kleiner werden, ist von bisher eher empirischen Nutzenbeurteilungen Abstand $\mathrm{zu}$ nehmen. Deswegen müssen und können in solchen Situationen aus ethischer und technischer Sicht methodisch konsistente Vergleichsstudien gefordert werden, welche auch eine Berechnung des Nutzens zulassen.

Heute fehlt in der Medizin vielerorts noch die Motivation, EBM und damit die Nutzenforschung zu fördern. Es wird befürchtet, bisherige Leistungen könnten durch die Versicherer gestrichen und damit das Terrain für eine Billigmedizin geebnet werden. Zudem fallen im heutigen Abgeltungssystem von medizinischen Einzelleistungen die Anreize klar zugunsten des Interventionismus aus, welcher ohnehin mehr Glaubwürdigkeit geniesst als die Erfassung der «Natural History».

Viele erwarten von den EBM-Spezialisten (z.B. in den Cochrane-Zentren), welche ihre Arbeit nicht am Patienten, sondern am Bürotisch verrichten, die Beantwortung der klinischen Fragestellungen und stützen sich nur noch auf deren Aussagen, ob Evidenz vorhanden ist oder nicht. Das Projekt «EBM Bottom up» der AWA zielt hingegen mehr auf die Praxisnähe ab. Die Ärzte sollen das nötige Rüstzeug erhalten, um bei Einzelentscheiden in konkreten Fällen die klinisch relevanten Fragen beantworten zu können.

Die AWA stützt sich mit ihrer Fortbildungsinitiative auf die Erkenntnis, dass Evidencebased Medicine ein hervorragendes Hilfsmittel darstellt, um die im naturwissenschaftlichen, eher mechanistischen Denken entstandenen Hypothesen auf ihre effektive biologische Auswirkung mit patientenrelevanten Endpunkten zu überprüfen. Zudem bleibt die AWA auch einem ihrer Leitsätze gerecht: die Medizin des Notwendigen zu fördern.

Informationen und Anmeldungsmöglichkeiten für die dreitägigen EBM-Kurse unter www. wig.ch. Winterthurer Institut für Gesundheitsökonomie WIG, Technopark/Jägerstrasse, Postfach 805, 8401 Winterthur, Tel. 0522677897 (Frau Eisenring), E-Mail: wig@zhwin.ch 